\title{
Photoacoustic lymphangiography before and after lymphaticovenular anastomosis
}

\author{
Anna $\mathrm{Oh}^{1}$, Hiroki Kajita ${ }^{1}$, Eri Matoba ${ }^{1}$, Keisuke Okabe ${ }^{1}$, Hisashi Sakuma ${ }^{2}$, Nobuaki Imanishi ${ }^{3}$, \\ Yoshifumi Takatsume ${ }^{3}$, Hikaru Kono ${ }^{1}$, Yasufumi Asao ${ }^{4}$, Takayuki Yagi ${ }^{4}$, Sadakazu Aiso ${ }^{3,4,5}$, \\ Kazuo Kishi ${ }^{1}$ \\ ${ }^{1}$ Department of Plastic and Reconstructive Surgery, Keio University School of Medicine, Tokyo; ${ }^{2}$ Department of Plastic and Reconstructive \\ Surgery, Yokohama Municipal Citizen's Hospital, Yokohama; ${ }^{3}$ Department of Anatomy, Keio University School of Medicine, Tokyo; ${ }^{4}$ Luxonus \\ Inc., Tokyo; ${ }^{5}$ SIT Research Laboratories, Shibaura Institute of Technology, Tokyo, Japan
}

Background Lymphaticovenular anastomosis (LVA) is a minimally invasive surgical procedure used to treat lymphedema. Volumetric measurements and quality-of-life assessments are often performed to assess the effectiveness of LVA, but there is no method that provides information regarding postoperative morphological changes in lymphatic vessels and veins after LVA. Photoacoustic lymphangiography (PAL) is an optical imaging technique that visualizes the distribution of light-absorbing molecules, such as hemoglobin or indocyanine green (ICG), and provides three-dimensional images of superficial lymphatic vessels and the venous system simultaneously. In this study, we performed PAL in lymphedema patients before and after LVA and compared the images to evaluate the effect of LVA.

Methods PAL was performed using the PAI-05 system in three patients (one man, two women) with lymphedema, including one primary case and two secondary cases, before LVA. ICG fluorescence lymphography was performed in all cases before PAL. Follow-up PAL was performed between 5 days and 5 months after LVA.

Results PAL enabled the simultaneous visualization of clear lymphatic vessels that could not be accurately seen with ICG fluorescence lymphography and veins. We were also able to observe and analyze morphological changes such as the width and the number of lymphatic vessels and veins during the follow-up PAL after LVA.

Conclusions By comparing preoperative and postoperative PAL images, it was possible to analyze the morphological changes in lymphatic vessels and veins that occurred after LVA. Our study suggests that PAL would be useful when assessing the effect of LVA surgery.

Keywords Photoacoustic lymphangiography / Lymphaticovenular anastomosis / Lymphedema
Correspondence: Anna Oh Department of Plastic and Reconstructive Surgery, Keio University School of Medicine, 35 Shinanomachi, Shinjuku, Tokyo 160-8582, Japan

Tel: $+81-3-3353-1211$

Fax: +81-3-3352-1054

E-mail:prsanna1006@gmail.com

Received: December 9, 2020 • Revised: January 10, $2021 \bullet$ Accepted: February 4, 2021

pISSN: 2234-6163 • elSSN: 2234-6171 • https://doi.org/10.5999/aps.2020.02404• Arch Plast Surg 2021;48:323-328

\section{INTRODUCTION}

Lymphedema is a chronic condition where excess lymphatic fluid collects in the interstitial tissues, followed by subsequent inflammation, fibrosis, and adipose tissue deposition. Lymphedema can occur as a result of a congenital defect or by a blockage in the lymphatic system, such as in cases where a lymph node becomes impaired or has been removed. Lymphaticoven- 
ular anastomosis (LVA), one of the surgical treatments for lymphedema, is a bypass procedure in which lymphatic vessels in the affected limb are directly connected to nearby veins for the purpose of redirecting excess lymphatic fluid into the venous system [1].

As a result of lymphedema treatment with LVA, subjective improvements of the affected area occurred in $70 \%-80 \%$ of cases, and objective improvements, as measured by volume changes, were observed in $40 \%-80 \%[2,3]$. Several methods are used to assess the efficacy of LVA, including indocyanine green (ICG) fluorescence lymphography, lymphoscintigraphy, magnetic resonance lymphography, and ultrasonography. However, the postoperative changes in the lymphatic system and veins after LVA remain largely unknown, as conventional imaging modalities have a limited ability to provide morphological information about the lymphatic vessels and veins.

Photoacoustic lymphangiography (PAL) is an imaging modality based on a photoacoustic technique, in which pulsed laser light is delivered into a light-absorbing tissue component. The delivered light energy is converted into heat, leading to transient expansion and thus ultrasonic emission. This expansion generates ultrasonic waves that are detected by the sensor and then the signals are processed to produce three-dimensional images. Thus, PAL can visualize the distribution of light-absorbing molecules, such as hemoglobin or ICG, and provide three-dimensional images of superficial lymphatic vessels and the venous system simultaneously [4]. Using PAL, we were able to obtain morphological information on the lymphatic vessels and veins in a single examination [5]. In this study, we compared PAL images before and after LVA to assess morphological changes in the lymphatic vessels and veins.

\section{METHODS}

PAL was performed in three patients enrolled from April 2018 to January 2019. ICG fluorescence lymphography was performed in all cases using a near-infrared camera system before PAL. The three-dimensional photoacoustic visualization system (PAI-05) was used for PAL [4]. ICG ( $5 \mathrm{mg} / \mathrm{mL}, 0.1 \mathrm{~mL}$ of ICG for each site) was administered subcutaneously at three sites of the affected foot of the patient, with two injections in the interdigital web of the dorsal aspect of the foot and one injection posterior to the lateral malleolus. The PAL examination was conducted at the medial and posterior sides of the lower limb. Depending on the findings of ICG fluorescence lymphography, the examination site was changed to where lymphatic vessels were observed. Postoperative PAL images were obtained at the same examination site where LVA had been performed before.
We analyzed the morphological characteristics of the lymphatic vessels and veins, such as their width and number, in both preoperative and postoperative PAL images, and then compared those images and analyzed their differences. The width of each lymphatic vessel was obtained by calculating the area from a transverse section taken from its maximum diameter (pi times the squared half of the transverse diameter).

All procedures were carried out in accordance with the ethical standards of the Ethics Committee at Keio University Hospital and the Certified Review Board of Keio (No. CRB3180017), and within the framework of the Helsinki Declaration of 1975, as revised in 1983. Patients were informed of the procedure to obtain their full collaboration and informed consent was obtained from all individual participants.

\section{RESULTS}

All patients underwent the PAL examinations without any complications. A linear and diffuse appearance of the lymphatic system was observed using ICG fluorescence lymphography of the affected extremity in all cases. It was difficult to identify each lymphatic vessel within the dermal backflow findings using ICG fluorescence lymphography. In contrast, with PAL, we were able to clearly observe lymphatic vessels that could not be seen accurately using ICG fluorescence lymphography. In preoperative PAL images, lymphatic vessels with a linear or tortuous appearance were identified in the affected limbs of all patients. In addition, PAL simultaneously provided information about veins. PAL was performed after LVA in all cases. When compared to preoperative PAL images, postoperative PAL showed various morphological changes in lymphatic vessels and veins.

\section{Case 1}

A 64-year-old woman presented with lymphedema of the left lower leg for 10 years after cervical cancer treatment. The patient underwent ICG fluorescence lymphography and PAL on the medial side of the left lower leg before and after LVA (Fig. 1A). Below the knee, the lymphatic vessels showed a linear appearance on ICG fluorescence lymphography. However, PAL identified five lymphatic collectors with a linear appearance that were not precisely seen with ICG fluorescence lymphography (Fig. 1B). The diameter of each lymphatic collector was 0.4-0.7 $\mathrm{mm}$. Postoperative PAL was performed 5 months after LVA (one side-to-end anastomosis). In the postoperative PAL examination (Fig. 1C), four additional lymphatic collectors that were assumed to be different pathways from the lymphatic collectors observed in the preoperative PAL image were observed at the medial aspect of the left lower leg. The diameter of each lym- 

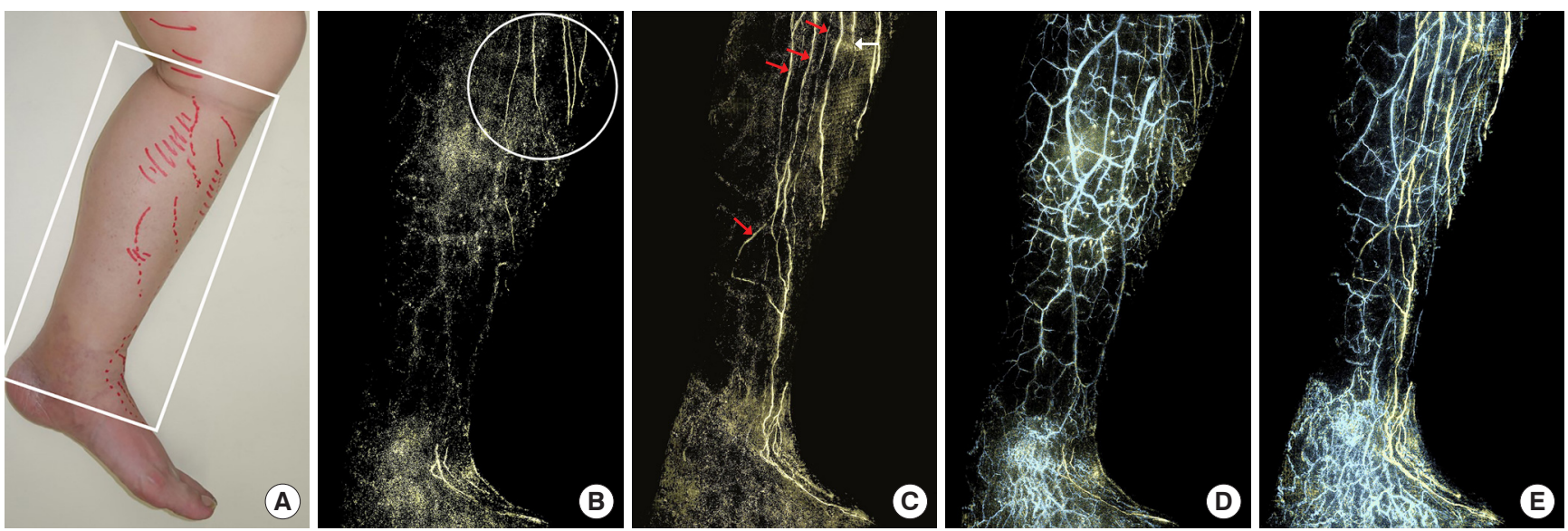

Fig. 1. Secondary lymphedema of the left leg. A 64-year-old woman presented with secondary lymphedema of the left leg. (A) An image of the medial side of the left lower leg shows the area examined preoperatively using indocyanine green (ICG) fluorescence lymphography and photoacoustic lymphangiography (PAL) (white square). The lymphatic vessels observed with ICG fluorescence lymphography were marked on the leg (red lines). (B) A preoperative PAL image shows the lymphatic vessels clearly (white circle). (C) A postoperative PAL image shows an increased number of dilated lymphatic vessels. Four lymphatic vessels, which ran in a pathway from the dorsal aspect of the foot to the proximal direction, were newly observed at the medial aspect of the left lower leg (red arrows). The bypassed lymphatic vessel (white arrow) was visualized from the LVA site to the proximal aspect. (D) Overlay of the preoperative PAL image shows lymphatic vessels (yellow) and veins (blue). (E) Overlay of the postoperative PAL image shows lymphatic vessels (yellow) and veins (blue).

phatic collector was $0.6-1.7 \mathrm{~mm}$. The lymphatic collectors observed in the preoperative PAL image appeared dilated by about 2.6 times in the affected leg after LVA. We were also able to observe that the lymphatic flow from the LVA site (Fig. 1C, white arrow) to the proximal aspect had built up in the bypassed lymphatic collector. The venous system was observed using PAL before LVA (Fig. 1D) and the width of the veins in the affected area appeared to have decreased after LVA (Fig. 1E). There was no significant difference in the number of veins observed before and after LVA.

\section{Case 2}

A 69-year-old woman was diagnosed with lymphedema of the right lower leg for 7 years after cervical cancer treatment. The patient underwent ICG fluorescence lymphography and PAL on the medial side of the right leg before and after LVA (Fig. 2A). Using ICG fluorescence lymphography, a linear appearance was observed in the affected leg. However, three lymphatic collectors were clearly identified using PAL (Fig. 2B). The patient underwent LVA at two sites: the distal (side-to-end anastomosis) and the proximal (end-to-end anastomosis) sides of the affected leg. Four additional lymphatic collectors were visible when PAL was performed 5 days after LVA (Fig. 2C). Two lymphatic collectors appeared to run in the same pathway that was observed in the preoperative PAL image, while on the posterior side of the affected leg, two other lymphatic collectors presented a different pathway. The diameters of the lymphatic collectors in the preoperative and postoperative PAL images were $0.6-1.0$ $\mathrm{mm}$ and $0.8-1.3 \mathrm{~mm}$, respectively. These lymphatic collectors were also dilated by about 1.5 times after LVA, as seen in case 1 . However, LVA did not significantly alter the veins of the affected leg, as shown by comparing the preoperative (Fig. 2D) and postoperative (Fig. 2E) PAL images in case 2.

\section{Case 3}

A 23-year-old man was diagnosed with primary lymphedema of both lower legs and underwent PAL and ICG fluorescence lymphography preoperatively. We only examined the right leg, as the symptomatic presentation (e.g., swelling) in the right leg was more severe than that in the left leg (Fig. 3A). Using ICG fluorescence lymphography, a diffuse appearance was observed at the distal part of the right leg. When PAL was performed preoperatively, two lymphatic collectors were observed (Fig. 3B). The diameters of the lymphatic collectors were about $0.3 \mathrm{~mm}$. The patient underwent LVA at three sites (one end-to-end anastomosis, two side-to-end anastomoses) on the distal side of the affected leg. PAL was then performed again 2 months after LVA and three lymphatic collectors were additionally observed at the posterior aspect of the affected leg, compared to the preoperative PAL image (Fig. 3C). The diameter of the lymphatic collector observed in the preoperative PAL image was also approximately $0.3 \mathrm{~mm}$ in the postoperative PAL image. There was no change in the diameter of the lymphatic collector in the PAL image after LVA. There were also no significant morphological changes in the veins (Fig. 3D and E). 

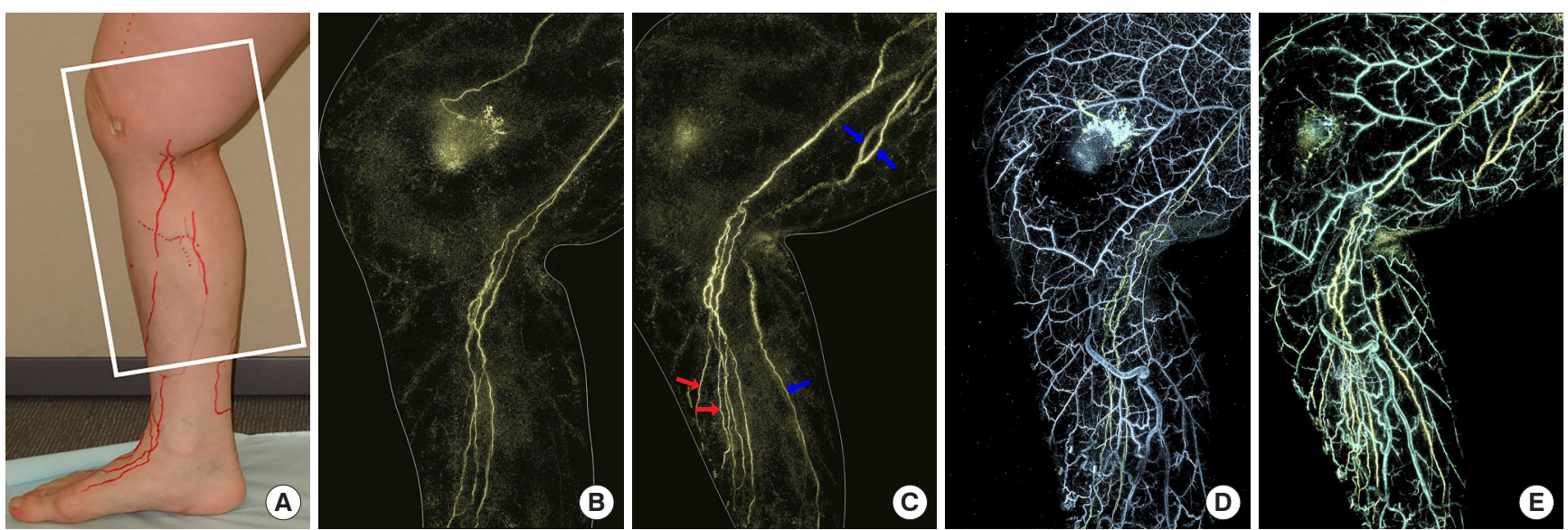

Fig. 2. Secondary lymphedema of the right leg. A 69-year-old woman presented with secondary lymphedema of the right leg. (A) An image of the medial side of the right lower leg shows the area examined using indocyanine green (ICG) fluorescence lymphography and photoacoustic lymphangiography (PAL) (white square). The lymphatic vessels observed with ICG fluorescence lymphography were marked on the leg (red lines). (B) A preoperative PAL image shows clear lymphatic vessels. (C) A postoperative PAL image shows additional four lymphatic vessels: two lymphatic vessels are observed on the posterior side of the affected leg (blue arrows), while the other two lymphatic vessels along run the same pathway with the lymphatic vessel observed in the preoperative PAL image (red arrows). (D) Overlay of the preoperative PAL image shows lymphatic vessels (yellow) and veins (blue). Adjacent veins are observed. (E) Overlay of the postoperative PAL image shows lymphatic vessels (yellow) and veins (blue).
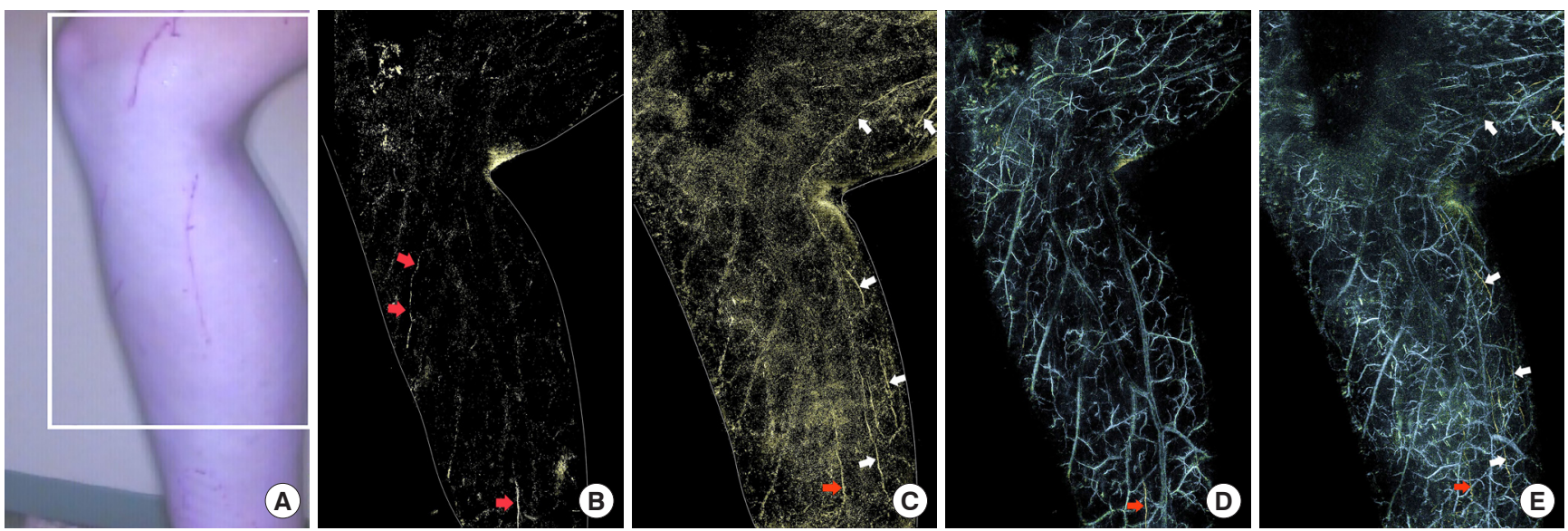

Fig. 3. Primary lymphedema of both legs. A 23-year-old man presented with primary lymphedema of the right leg. (A) An image of the medial side of the right lower leg shows the area examined using indocyanine green (ICG) fluorescence lymphography and photoacoustic lymphangiography (PAL) (white square). (B) A preoperative PAL image shows two lymphatic vessels (red arrows). (C) A postoperative PAL image shows an increased number of lymphatic vessels on the posterior side of the affected leg (white arrows). There is no remarkable change in the width of the lymphatic vessels (red arrow). (D) Overlay of the preoperative PAL image shows lymphatic vessels (yellow: red arrow) and veins (blue). Adjacent veins are visualized. (E) Overlay of the postoperative PAL image shows lymphatic vessels (yellow: red arrow and white arrows) and veins (blue).

\section{DISCUSSION}

The primary function of the lymphatic system is reabsorption and transportation of interstitial fluid that accumulates in the interstitial tissue space [6]. Lymphedema is a chronic disease caused by compromised lymphatic drainage. Regarding the pathological manifestations of lymphedema, the primary change is the accumulation of interstitial fluid due to insufficient lymphatic drainage, which leads to an increase in the tissue osmotic pressure and inflammatory changes $[4,7]$. Stasis of the in- terstitial fluid results in outflow resistance and an increase in lymphatic pressure, which induces lymphatic vessel dilation and valve dysfunction [8]. As lymphedema progresses, fibrotic changes occur in the lymphatic vessels due to collagen deposition and the lymphatic vessels become contracted and lose their normal permeability $[9,10]$. Adipose tissue deposition is the final pathological feature of lymphedema, and results from tissue fibrosis and chronic inflammation [11]. Therefore, management of tissue fluid accumulation in the early stage can be effective for preventing the development of subsequent pathological 
changes and lymphedema progression.

However, the exact pathophysiology of lymphedema is still unclear; therefore, no consensus exists regarding the most appropriate treatment $[8,12]$. Understanding the pathophysiology of lymphedema and the morphological changes of the lymphatic vessels according to the extent of disease progression is important for the successful treatment of lymphedema.

LVA is a surgical method used for the treatment of lymphedema. Impaired lymphatic vessels are anastomosed to adjacent veins, and improvement in lymphatic drainage occurs by allowing excess lymph to bypass areas of low or obstructed lymph flow [4]. Volumetric measurements and quality-of-life assessments are commonly used to assess the effects of LVA. However, no method directly provides sufficient evidence of the effect of LVA on lymphedema. Various imaging methods, such as computed tomography scans, magnetic resonance lymphography, and ICG fluorescence lymphography, are used; however, these modalities have limitations in their ability to describe the relevant physiological and morphological changes, including the flow, distribution, number, and subtle structure of the lymphatic vessels and veins.

PAL enables a comparison of the morphological changes of the lymphatic vessels and veins as a result of LVA. Below, we present a potential explanation of our results regarding morphological changes within the lymphatic vessels and veins after LVA, based on current knowledge on the pathophysiology of lymphedema.

In our study, PAL demonstrated an increase in the number of lymphatic collectors seen in the affected areas of patients after LVA treatment. The new lymphatic collectors shown in postoperative PAL images most likely were not regenerated, but instead became visible owing to a reduction in the distance between the surface of the skin and the lymphatic collectors concomitantly with a reduction in the severity of edema. The PAL device has a depth range of up to about $2 \mathrm{~cm}$; thus, as edema decreases, the ability of PAL to visualize vessels within the deeper layer improves. In case 1 , the lymphatic collectors that were newly revealed by postoperative PAL constituted pathways that led from the dorsal aspect of the foot and continued to the proximal side of the leg. When comparing before-and-after PAL images, we observed an increased number of lymphatic collectors, which may reflect the reduction in the swelling of the affected area.

The diameters of the lymphatic collectors in the postoperative PAL images were also significantly wider than those in preoperative PAL images in cases 1 and 2. In lymphedema, lymphatic vessels are initially dilated owing to increased lymphatic pressure, but they become contracted due to wall thickening as lymphedema progresses [13]. A change in the diameter of a lymphatic vessel can reflect disease progression according to the above-described pathophysiology of lymphedema. It is known that lymphatic dilation is reduced due to decreased interstitial pressure when lymphedema improves. However, it remains unclear what changes actually occur in the diameter of lymphatic vessels after edema is reduced. Either dilation or contraction of lymphatic vessels may appear during the process of edema reduction because the diameter of lymphatic vessels is affected by several factors, including lymphatic fibrosis, interstitial fluid pressure, and lymphatic flow $[12,14]$.

The outcomes of cases 1 and 2 were likely caused by an increased flow of lymphatic fluid or improvement of lymphatic collector contraction, suggesting that the wall thickening was reduced after LVA, or that both of the above-discussed mechanisms may have been involved. In addition, it is possible that these changes may have resulted from an improvement of lymphatic drainage.

In contrast, in case 3 (primary lymphedema), no change in the lymphatic collector diameter was observed after LVA. However, clinical manifestations such as edema had reduced. PAL has limitations in imaging lymphatic vessels located in the deep layer, so this result suggests only that the lymphatic collectors observed in the preoperative PAL image were not changed by LVA.

Regarding the effect of LVA on the veins of the affected limb, only one patient (case 1) presented thinner-appearing veins as shown by PAL. In lymphedema, the vascular system increases blood flow through vasodilatation and angiogenesis to compensate for impaired lymphatic drainage, which leads to an increased filtration load $[8,14]$. We suggest that the reduced diameter of the vein after LVA was caused by a decrease in the venous pressure as lymphatic drainage improved, followed by decreased interstitial fluid pressure.

A limitation of this study is that the number of patients was small. Further studies need to be done to establish the morphological changes of lymphatic vessels and veins after LVA.

PAL enabled a detailed comparison of the morphological changes of the lymphatic collectors and veins in individuals with lymphedema before and after LVA. The obtained results objectively document the effects of LVA, and may also provide information about the degree of lymphatic progression, which would be useful for selecting patients for LVA and predicting lymphedema improvement.

\section{NOTES}

\section{Conflict of interest}

Anna Oh, Hiroki Kajita, Nobuaki Imanishi, Hikaru Kono, Yasu- 
fumi Asao, Takayuki Yagi, Sadakazu Aiso, and Kazuo Kishi report grants from the ImPACT Program of Council for Science, Technology and Innovation (Cabinet Office, Government of Japan), during the conduct of the study. The other authors report no conflict of interest.

\section{Ethical approval}

The study was approved by the Ethics Committee at Keio University Hospital and the Certified Review Board of Keio (approval No. CRB3180017), and performed in accordance with the principles of the Declaration of Helsinki. Written informed consent was obtained.

\section{Patient consent}

The patients provided written informed consent for the publication and the use of their images.

\section{Author contribution}

Conceptualization: A Oh, H Kajita, N Imanishi. Data curation: A Oh, E Matoba, K Okabe, Y Takatsume. Formal analysis: A Oh, H Kajita, E Matoba, H Sakuma, N Imanishi. Funding acquisition: A Oh, H Kajita, S Aiso, K Kishi. Project administration: H Kajita, N Imanishi, Y Asao, T Yagi, S Aiso, K Kishi. Visualization: A Oh, Y Takatsume, H Kono, Y Asao, T Yagi. Writing - original draft: A Oh. Writing - review \& editing: A Oh, H Kajita, E Matoba, K Okabe, H Sakuma, N Imanishi, Y Takatsume, Y Asao, T Yagi, S Aiso, K Kishi.

\section{ORCID}

$\begin{array}{lr}\text { Anna Oh } & \text { https://orcid.org/0000-0003-1422-2205 } \\ \text { Hiroki Kajita } & \text { https://orcid.org/0000-0002-3267-5884 } \\ \text { Eri Matoba } & \text { https://orcid.org/0000-0003-2322-6208 } \\ \text { Keisuke Okabe } & \text { https://orcid.org/0000-0001-8614-5195 } \\ \text { Hisashi Sakuma } & \text { https://orcid.org/0000-0002-6073-6832 } \\ \text { Nobuaki Imanishi } & \text { https://orcid.org/0000-0002-8816-6309 } \\ \text { Yoshifumi Takatsume https://orcid.org/0000-0002-9467-2021 } \\ \text { Hikaru Kono } & \text { https://orcid.org/0000-0001-5736-0312 } \\ \text { Yasufumi Asao } & \text { https://orcid.org/0000-0002-9757-809X } \\ \text { Takayuki Yagi } & \text { https://orcid.org/0000-0002-4396-4745 } \\ \text { Sadakazu Aiso } & \text { https://orcid.org/0000-0001-8867-5237 } \\ \text { Kazuo Kishi } & \text { https://orcid.org/0000-0002-4298-9828 }\end{array}$

\section{REFERENCES}

1. Olszewski WL. The treatment of lymphedema of the extremities with microsurgical lympho-venous anastomoses. Int Angiol 1988;7:312-21.

2. Campisi C, Boccardo F, Zilli A, et al. Long-term results after lymphatic-venous anastomoses for the treatment of obstructive lymphedema. Microsurgery 2001;21:135-9.

3. O’Brien BM, Mellow CG, Khazanchi RK, et al. Long-term results after microlymphaticovenous anastomoses for the treatment of obstructive lymphedema. Plast Reconstr Surg 1990;85:562-72.

4. Nagae K, Asao Y, Sudo Y, et al. Real-time 3D photoacoustic visualization system with a wide field of view for imaging human limbs. F1000Res 2018;7:1813.

5. Kajita H, Oh A, Urano M, et al. Photoacoustic lymphangiography. J Surg Oncol 2020;121:48-50.

6. Jiang X, Nicolls MR, Tian W, et al. Lymphatic dysfunction, leukotrienes, and lymphedema. Annu Rev Physiol 2018;80: 49-70.

7. Grada AA, Phillips TJ. Lymphedema: pathophysiology and clinical manifestations. J Am Acad Dermatol 2017;77:100920.

8. Mortimer PS. The pathophysiology of lymphedema. Cancer 1998;83(12 Suppl American):2798-802.

9. Azhar SH, Lim HY, Tan BK, et al. The unresolved pathophysiology of lymphedema. Front Physiol 2020;11:137.

10. Rockson SG. Lymphedema. Am J Med 2001;110:288-95.

11. Cucchi F, Rossmeislova L, Simonsen L, et al. A vicious circle in chronic lymphoedema pathophysiology? An adipocentric view. Obes Rev 2017;18:1159-69.

12. Hespe GE, Nores GG, Huang JJ, et al. Pathophysiology of lymphedema: is there a chance for medication treatment? J Surg Oncol 2017;115:96-8.

13. Barone V, Borghini A, Tedone Clemente E, et al. New insights into the pathophysiology of primary and secondary lymphedema: histopathological studies on human lymphatic collecting vessels. Lymphat Res Biol 2020;18:502-9.

14. Himeno Y, Ikebuchi M, Maeda A, et al. Mechanisms underlying the volume regulation of interstitial fluid by capillaries: a simulation study. Integr Med Res 2016;5:11-21. 